\title{
The POD-TPWL Method of Accelerating Reservoir Numerical Simulation
}

\author{
CAO Jing \\ (School of Information and Mathematics, Yangtze University, Jingzhou,434023, China)
}

\begin{abstract}
Improving reservoir simulation computing speed is of great significance under the existing calculation conditions. Model order reduction (MOR) technique represents a promising approach for accelerating the simulations. In this work, we combine proper orthogonal decomposition (POD) and trajectory piecewise-linear (TPWL) method. This method is called POD-TPWL method. First, the nonlinear system is represented as a weighted combined piecewise linear system using TPWL method, and then reducing order of each linear model using POD. We apply POD-TPWL method for a two-phase (oil-water) reservoir model which is solved by full implicit. The example demonstrates that which can greatly reduce the dimension of reservoir model, so as to reduce the calculation time and improve the operation speed.
\end{abstract}

Keywords: reservoir simulation; model order reduction; trajectory piecewise-linear; proper orthogonal decomposition

\section{Introduction}

Numerical reservoir simulation is very important for modern reservoir management. Traditional reservoir simulators numerically solve a set of governing partial differential equations. This entails solving a set of nonlinear algebraic equations by using iteration. These numerical solutions can be quite time consuming, however, because the reservoir simulation models arising from real fields may consist of hundreds of thousands or millions of grid blocks and the number of equations that must be solved can be very large [1]. Therefore, in the case of ensuring the sufficient accuracy of numerical solution, how to greatly accelerate the reservoir simulation speed is the urgent problem to be solved.

Model order reduction (MOR) is the transformation of high-dimensional models into meaningful representations of reduced dimensionality. It has shown promise in alleviating computational demands with minimal loss of accuracy [2]. The proper orthogonal decomposition method (POD) is the most widely used in nonlinear system model reduction method. For now, POD is also widely applied to reservoir simulation [3-5]. Although the POD method can be applied to the nonlinear reservoir simulation system, the acceleration is limited, because in the simulation process, each iteration step requires the construction and projection of the full order Jacobian matrix. At present, the trajectory piecewise-linear (TPWL) [6] reduced order method is be widely used in nonlinear system. The nonlinear system is represented as a weighted combined piecewise linear system. In this paper, the TPWL model reduction method is applied to reservoir simulator, and then reducing order of each linear model using POD method. This method is called POD-TPWL. It can greatly reduce the dimension of reservoir model, so as to reduce the calculation time and improve the operation speed.

\section{Reservoir Model}

In this paper, the mathematical model of reservoir model is transformed into the state space equation by means of space discrete in order to explain the reduction process of TPWL method. Two dimensional oil-water two phase reservoir model is used. It is assumed that oil and water do not exchange material, the process is isothermal, the fluid is compressible, and the mass conservation equation and Darcy's law can be used to obtain [7]:

$$
-\nabla \bullet\left[\frac{k_{r i} \rho_{i}}{\mu_{i}} \mathbf{K}\left(\nabla p_{i}-\rho_{i} g \nabla d\right)\right]+\frac{\partial\left(\phi S_{i} \rho_{i}\right)}{\partial t}-\rho_{i} q_{i}^{\prime \prime \prime}=0
$$

Where $\mathbf{K}$ is permeability tensor; $\mu$ is fluid viscosity; $k_{r}$ is relative permeability; $p$ is pressure; $g$ is gravity acceleration; $d$ is depth; fluid density; $\phi$ is porosity; $S$ is fluid saturation; $t$ is time; $q$ "' is a source term expressed as flow rate per unit volume; superscript $i \in\{o, w\}$ is respectively oil phase and water phase. In the equation (1), there are four unknown quantities, $p_{w}$ and $S_{o}$ are eliminated by using the auxiliary equation (2) and (3), so that only the state variables $p_{o}, S_{w}$ are included in the equation, 


$$
\begin{gathered}
S_{o}+S_{w}=1 \\
p_{o}-p_{w}=p_{c}\left(S_{w}\right)
\end{gathered}
$$

Where $p_{c}\left(S_{w}\right)$ is oil-water two-phase capillary pressure.

We consider the relatively simple cases and ignore gravity and capillary force. Format to discrete in space by using five point block centered finite difference, we may have the nonlinear first-order differential equation (4), see the specific derivation of literature [8]:

$$
\underbrace{\left[\begin{array}{ll}
\mathbf{V}_{w p} & \mathbf{V}_{w s} \\
\mathbf{V}_{o p} & \mathbf{V}_{o s}
\end{array}\right]}_{\mathbf{V}}\left[\begin{array}{c}
\dot{\mathbf{p}} \\
\dot{\mathbf{s}}
\end{array}\right]+\underbrace{\left[\begin{array}{cc}
\mathbf{T}_{w} & \mathbf{0} \\
\mathbf{T}_{o} & \mathbf{0}
\end{array}\right]}_{\mathbf{T}}\left[\begin{array}{c}
\mathbf{p} \\
\mathbf{s}
\end{array}\right]=\underbrace{\left[\begin{array}{c}
\mathbf{F}_{w}(\mathbf{s}) \\
\mathbf{F}_{o}(\mathbf{s})
\end{array}\right]}_{\mathbf{F}} \mathbf{q}_{w e l l, t}
$$

Where: vector $\mathbf{p}$ and $\mathbf{S}$ is grid center oil pressure $p_{o}$ and water saturation $S_{w}$ respectively; $\dot{\mathbf{p}}$ and $\dot{\mathbf{S}}$ is the time $t$ derivative of vector $\mathbf{p}$ and $\mathbf{S}$ respectively; $\mathbf{V}$ is the cumulative matrix; $\mathbf{T}$ is transmission matrix; $\mathbf{F}$ is divided flow matrix; Vector $\mathbf{q}_{\text {well }, t}$ is the total flow of oil-water well.

Define the state vector $\mathbf{X}$, input vector $\mathbf{u}$ and output vector $\mathbf{y}$

$$
\mathbf{x} \square\left[\begin{array}{l}
\mathbf{p} \\
\mathbf{s}
\end{array}\right] \quad \mathbf{u} \square\left[\begin{array}{l}
\breve{\mathbf{q}}_{\text {well }, t} \\
\breve{\mathbf{p}}_{\text {well }}
\end{array}\right] \quad \mathbf{y} \square\left[\begin{array}{l}
\overline{\mathbf{p}}_{\text {well }} \\
\overline{\mathbf{q}}_{\text {well }, w} \\
\overline{\mathbf{q}}_{\text {well }, \mathrm{o}}
\end{array}\right]
$$

Where vector $\breve{\mathbf{q}}_{\text {well }, t}$ and $\breve{\mathbf{p}}_{\text {well }}$ represent the well of the constant flow and the bottom hole pressure respectively; The vector $\overline{\mathbf{p}}_{\text {well }}$ indicates the output bottom hole flow pressure of the constant flow well; Vector $\overline{\mathbf{q}}_{\text {well }, \mathrm{o}}$ and $\overline{\mathbf{q}}_{\text {well, },}$ indicate the output oil and water flow of the constant bottom hole pressure respectively. The equation (4) can be written as the form of state space equation [8]:

$$
\begin{aligned}
\dot{\mathbf{x}} & =\mathbf{f}(\mathbf{x}, \mathbf{u})=\mathbf{A}(\mathbf{x}) \mathbf{x}+\mathbf{B}(\mathbf{x}) \mathbf{u} \\
\mathbf{y} & =\mathbf{h}(\mathbf{x}, \mathbf{u})=\mathbf{C}(\mathbf{x}) \mathbf{x}+
\end{aligned}
$$

In the control system, $\mathbf{A}$ is called the system matrix, $\mathbf{B}$ is called the input matrix, $\mathbf{C}$ is called the output matrix, $\mathbf{D}$ is called the direct transfer matrix. Because the elements of the matrix $\mathbf{V}, \mathbf{T}, \mathbf{F}, \mathbf{J}$ are function of the state variables, the system is a nonlinear system.

\section{POD-TPWL Reduced Order Method}

In order to construct a POD basis vector, the first we need to run a full order reservoir simulator (also known as the training process), and to preserve the state vector $\mathbf{X}$ of each time step (also called the snapshot, including the oil pressure $p_{o}$ and the water saturation $S_{w}$ of all grids). Because of pressure and saturation with different physical properties, we use matrix $\mathbf{X}_{p}, \mathbf{X}_{S}$ to preserve $p_{o}, S_{w}$ respectively (hereinafter abbreviated as $p$ and $S$ ):

$$
\mathbf{X}_{p}=\left[\begin{array}{llll}
\mathbf{x}_{p}{ }^{1} & \mathbf{x}_{p}{ }^{2} \cdots & \mathbf{x}_{p}{ }^{m}
\end{array}\right], \quad \mathbf{X}_{S}=\left[\begin{array}{llll}
\mathbf{x}_{S}{ }^{1} & \mathbf{x}_{S}{ }^{2} \cdots & \mathbf{x}_{S}{ }^{m}
\end{array}\right]
$$

Assuming that the number of grids in the reservoir model is $N$, then each vector $\mathbf{x}_{p}{ }^{i}, \mathbf{x}_{S}{ }^{i}$ (Superscript $i$ denotes the number of snapshots)in the matrix $\mathbf{X}_{p}$ and $\mathbf{X}_{S}$ is $N$ dimension, however, the dimension of the state vector $\mathbf{X}$ of the system is: $n=2 N$. The snapshot needs to be calculated mean value after the snapshot is obtained:

$$
\overline{\mathbf{x}}_{p}=\frac{1}{m} \sum_{i=1}^{m} \mathbf{x}_{p}{ }^{i}, \quad \overline{\mathbf{x}}_{S}=\frac{1}{m} \sum_{i=1}^{m} \mathbf{x}_{S}{ }^{i}
$$

And each snapshot in the data matrix $\mathbf{X}_{p}$ and $\mathbf{X}_{S}$ is subtracted from the mean:

$$
\hat{\mathbf{X}}_{p}=\left[\mathbf{x}_{p}{ }^{1}-\overline{\mathbf{x}}_{p}, \mathbf{x}_{p}{ }^{2}-\overline{\mathbf{x}}_{p}, \cdots, \mathbf{x}_{p}{ }^{m}-\overline{\mathbf{x}}_{p}\right]
$$




$$
\hat{\mathbf{X}}_{S}=\left[\mathbf{x}_{S}{ }^{1}-\overline{\mathbf{x}}_{S}, \mathbf{x}_{S}{ }^{2}-\overline{\mathbf{x}}_{S}, \cdots, \mathbf{x}_{S}{ }^{m}-\overline{\mathbf{x}}_{S}\right]
$$

Implementing the above POD reduction process for the matrix $\hat{\mathbf{X}}_{p}$, the basis vector matrix $\boldsymbol{\Phi}_{l p}$ and $\boldsymbol{\Phi}_{l s}$ are obtained, and we combine the two matrices to obtain basis matrix $\boldsymbol{\Phi}_{l}$, which includes $l$ columns, and $l=l_{p}+l_{s}$.

By using the TPWL method, a set of linearized points is obtained by using a kind of linear expansion point selection algorithm: $\hat{\mathbf{x}}_{0}, \hat{\mathbf{x}}_{1}, \cdots, \hat{\mathbf{x}}_{s-1}$. Near the linearization points, a set of linear models are obtained by the linear expansion of the nonlinear term $\mathbf{f}(\mathbf{x})=\mathbf{A}(\mathbf{x}) \mathbf{x}$ :

$$
\dot{\mathbf{x}}=\mathbf{G}_{\mathbf{i}} \mathbf{x}+\left(\mathbf{f}\left(\hat{\mathbf{x}}_{\mathbf{i}}\right)-\mathbf{G}_{\mathbf{i}} \hat{\mathbf{x}}_{\mathbf{i}}\right)+\mathbf{B}_{i} \mathbf{u}, \quad i=0,1, \cdots,(\mathrm{s}-1)
$$

Where: $\mathbf{G}_{\mathbf{i}}$ is Jacobian matrix of $\mathbf{f}(\mathbf{x})$ at $\hat{\mathbf{x}}_{\mathbf{i}}, \mathbf{B}_{i}=\mathbf{B}\left(\hat{\mathbf{x}}_{i}\right)$.

By using weighted function, the approximate reduction system of the nonlinear system (8) is obtained by weighted summation of the formula (13)

$$
\dot{\mathbf{x}}=\sum_{i=0}^{s-1} \omega_{i}(\mathbf{x})\left(\mathbf{G}_{i} \mathbf{x}+\left(\mathbf{f}\left(\hat{\mathbf{x}}_{\mathbf{i}}\right)-\mathbf{G}_{\mathbf{i}} \hat{\mathbf{x}}_{\mathbf{i}}\right)+\mathbf{B}_{i} \mathbf{u}\right), \quad i=0,1,, \quad,+\mathrm{s}
$$

Setting $\mathbf{X} \approx \Phi_{l} \mathbf{z}$, we can get the approximation of nonlinear system (8), (9) for order reduction system

$$
\begin{gathered}
\dot{\mathbf{z}}=\sum_{i=0}^{s-1} \omega_{i}(\mathbf{z})\left(\mathbf{G}_{i r} \mathbf{z}+\mathbf{V}^{T}\left(\mathbf{f}\left(\hat{\mathbf{x}}_{\mathbf{i}}\right)-\mathbf{G}_{\mathbf{i}} \hat{\mathbf{x}}_{\mathbf{i}}\right)+\mathbf{B}_{i r} \mathbf{u}\right) \\
\mathbf{y}=\mathbf{C}_{r} \mathbf{z}+\mathbf{D u}
\end{gathered}
$$

In the literature [6], the proposed algorithm for generating the collection of linearized models may be summarized in the following steps:

1) Generate a linearized model about the initial state $\hat{\mathbf{x}}_{0}=\mathbf{x}_{0}$, and set $i=0$

2) Simulate the nonlinear system while $\min _{0 \leq j \leq i}\left\|\mathbf{x}-\mathbf{x}_{j}\right\|>\delta$ for some $\delta>0$,

i.e. while the current state $\mathrm{x}$ is close enough to any of the previous linearization points;

3) Generate a new linearized model about $\hat{\mathbf{x}}_{i+1}=\mathbf{x}$, and set $i:=i+1$

4) If $i<s-1$, return to step 2 .

In the literature [6], the calculation of the weight function $\omega_{i}(\mathbf{z})$ of the current state $\mathbf{Z}$ is as follows:

1) For $i=0,1, \cdots,(s-1)$ compute $d_{i}=\left\|\mathbf{z}-\hat{\mathbf{z}}_{i}\right\|_{2}$

2) Take $m=\min _{i=0, \cdots,(\mathrm{s}-1)} d_{i}$

3) For $i=0,1, \cdots,(s-1)$ compute $\hat{\omega}_{i i}=e^{-\beta d_{i} / m}$, take $\beta=25$

4) Normalize $\hat{\omega}_{i}$ at the evaluation point:

a) compute $S(\mathbf{z})=\sum_{j=0}^{s-1} \hat{\omega}_{j}(\mathbf{z})$;

b) For $i=0,1, \cdots,(s-1)$, set $\omega_{i}(\mathbf{z})=\hat{\omega}_{i}(\mathbf{z}) / S(\mathbf{z})$.

\section{Example Verification}

The numerical example is that a two-dimensional oil-water two phase anisotropic reservoir is described. Its grid is divided into $22 * 22$, and the distribution of permeability and porosity is shown in Figure 1,2 . The related parameters of reservoir model: thickness $\mathrm{h}=3 \mathrm{~m}$, length and width of grid $\Delta x=\Delta y=33.33 \mathrm{~m}$, the viscosity of the crude oil $\mu_{o}=5 \mathrm{mPa} \cdot \mathrm{s}$, formation water viscosity $\mu_{w}=1 \mathrm{mPa} \cdot \mathrm{s}$, comprehensive compression coefficient $c_{t}=3.0 \times 10^{-3} \mathrm{MPa}^{-1}$, the original formation pressure $p_{i}=30 \mathrm{MPa}$, borehole radius $r_{\text {well }}=0.114 \mathrm{~m}$, the end point relative permeability of oil phase $k_{r o}^{0}=0.9$, the end point relative permeability of water phase 
$k_{r w}^{0}=0.6$, oil phase Corey index $n_{o}=2.0$, water phase Corey index $n_{w}=2.0$, residual oil saturation $S_{o r}=0.2$, irreducible water saturation $S_{w c}=0.2$. We use anti five point method well pattern to produce. Center has a water injection well, and four corners have four production wells. We ignore gravity and capillary force.
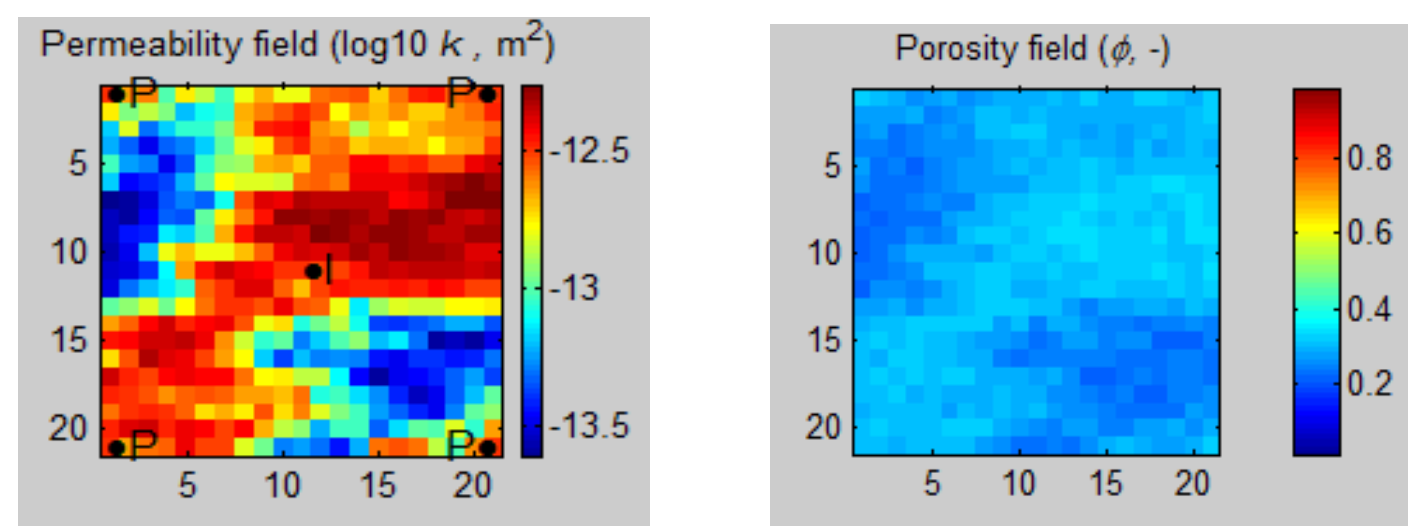

Fig.1 Permeability distribution of reservoir model Fig.2 Porosity distribution of reservoir model The numerical example is simulated by a fully implicit processing. It is divided into training and forecasting two processes:

\section{(1) Training process}

The bottom hole pressure of production well is $26.5 \mathrm{MPa}$, the bottom hole flow of injection well is $0.0015 \mathrm{~m}^{3} / \mathrm{s}$. We run the full order simulator for 1400 days and save the results of the 66 time steps. The pressure matrix retains 32 singular values, saturation matrix retains 34 singular values, and the dimension of the base matrix $\Phi_{l}$ of POD is $2 N \times l$, of which $l=32+34=66$. This means that the full order simulator is required to solve $2 N=882$ unknown variable, while the reduced order only needs to solve 66 variables. The number of selected linearization point is 11 .

In the training process, the comparison between the full order reservoir simulator and the reduced order simulator using TPWL method is shown in figure 3, 4 .

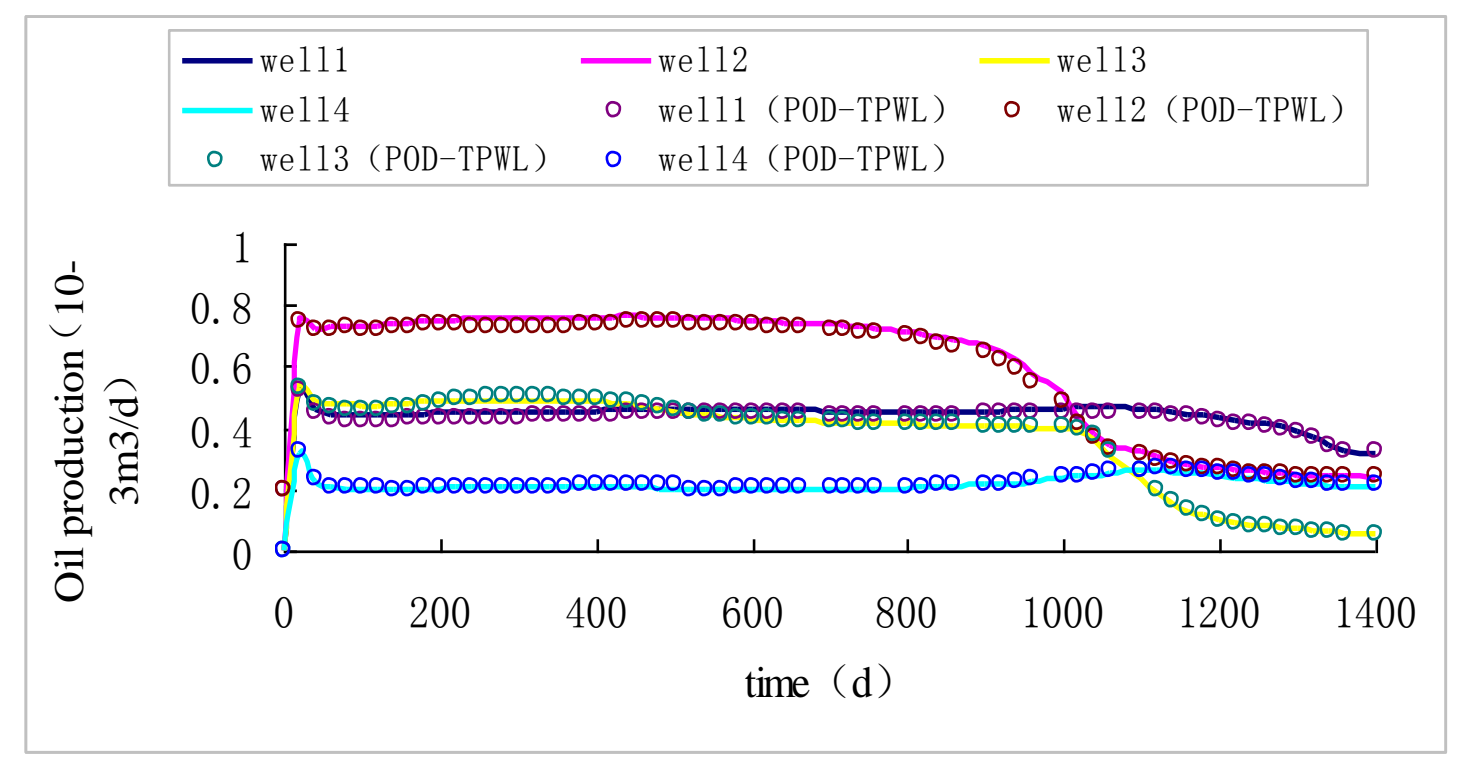

Fig.3 Oil production contrast of four production wells (training process) 


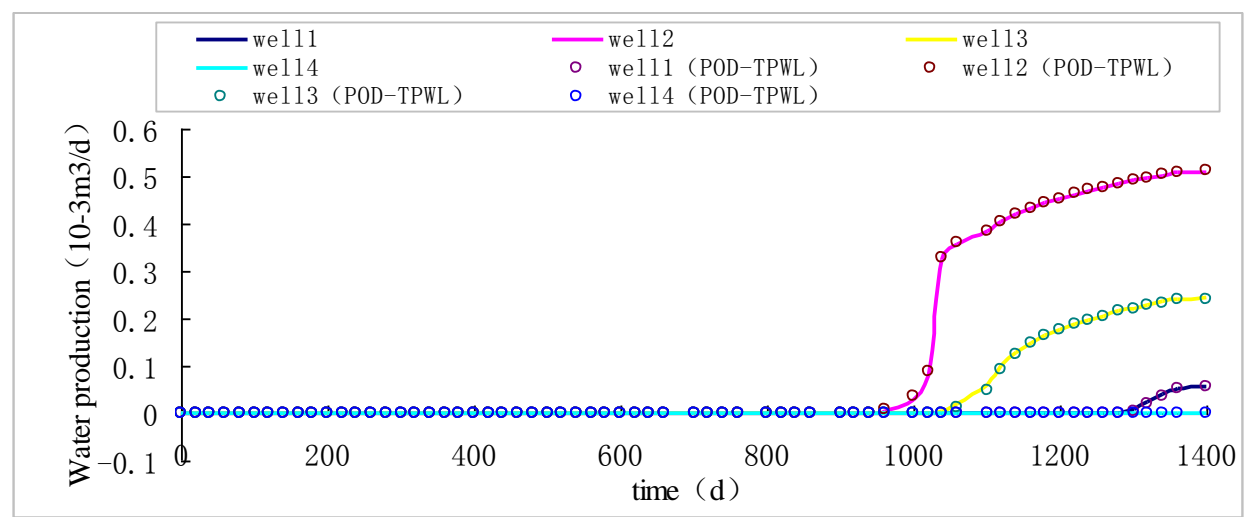

Fig.4 Water production contrast of four production wells (training process)

The above results indicate that in the training process, oil production and water production of four production wells of reduce order and full order simulator are almost identicall, but the simulation time is increased nearly 10 times, the running time of the full order simulator is $35.866 \mathrm{~s}$, and the running time of reduction simulator is $3.612 \mathrm{~s}$.

\section{(2) Forecasting process}

At this time, the bottom hole pressure of production wells is changed to $25.5 \mathrm{MPa}$, and the flow rate at the bottom of the injection well remains unchanged. The comparison between the full order simulator and the reduced order simulator is shown in figure 5, 6 .

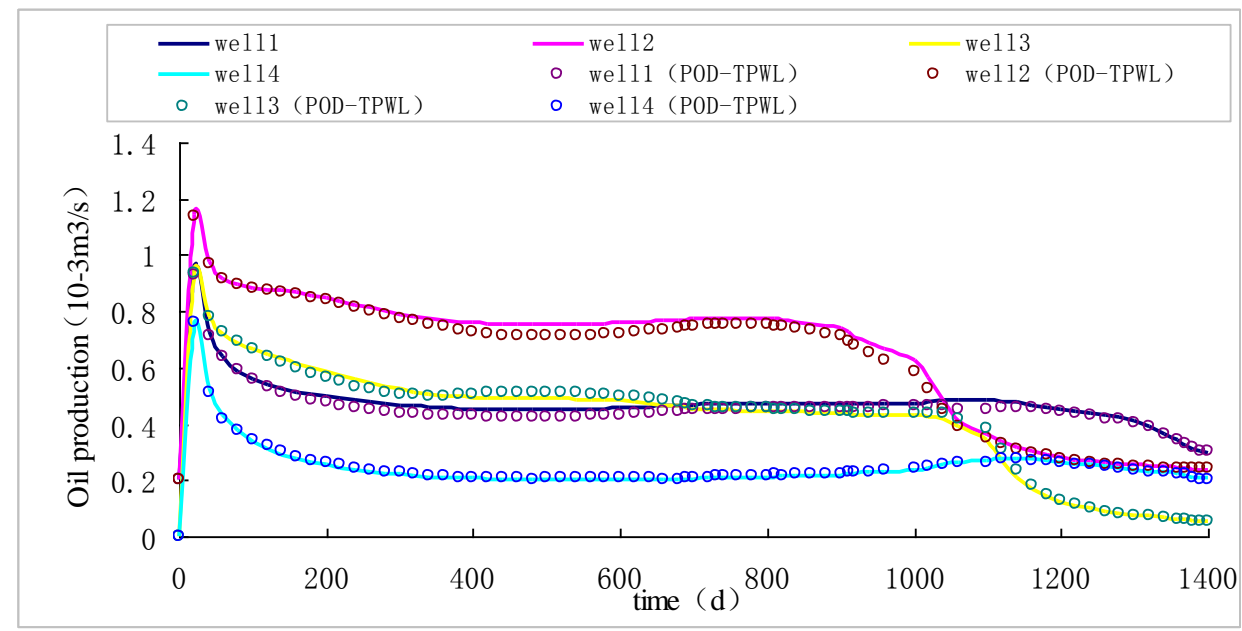

Fig. 5 Oil production contrast of four production wells (prediction process)

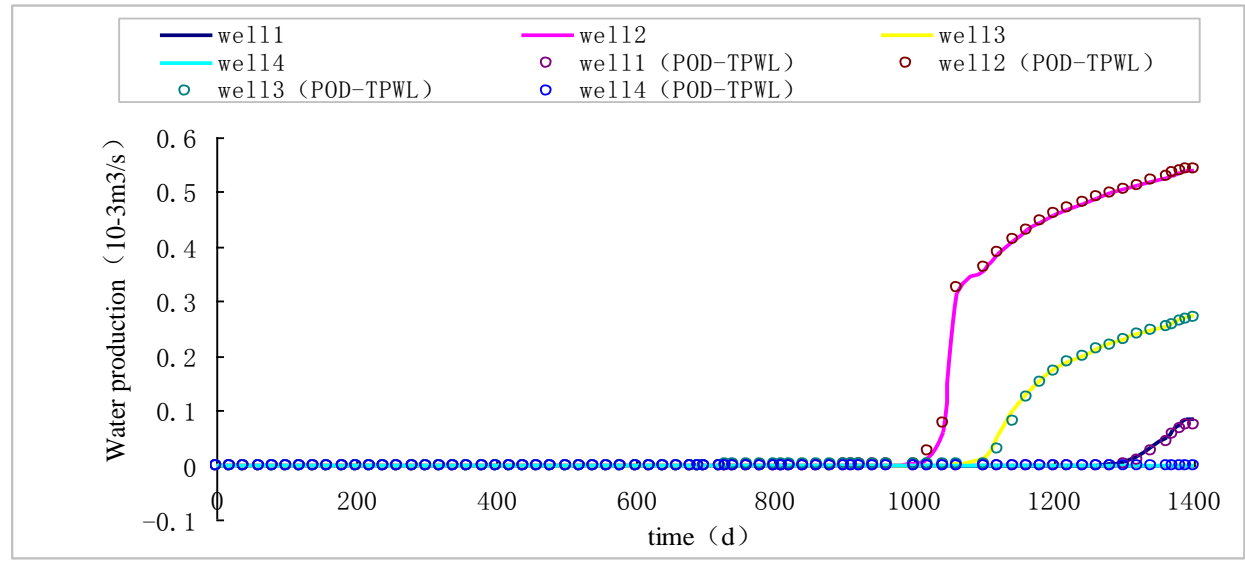

Fig. 6 Water production contrast of four production wells (prediction process) 
In the forecasting process, the results show that when the production schedule of forecasting process and training process are different, oil production and water production of four production wells of reduce order and full order simulator are also almost identicall. The full order simulator runs for $35.446 \mathrm{~s}$, and the running time of the reduced order simulator is $3.601 \mathrm{~s}$.

\section{Conclusion}

1) The application of POD-TPWL model reduced order method to reservoir simulator can greatly reduce the dimension of reservoir model, and improve the operation speed of the simulator by nearly 9 times.

2) When the production schedule of the training and forecasting process is different, oil production and water production of four production wells of reduce order and full order simulator are also almost identicall.

3) The improvement of the operation speed of the reservoir simulator provides an important solution for the practical application of the reservoir production optimization and history matching.

\section{Reference}

[1]. J. He.Reduced-order modeling for oil-water and compositional systems, with application to data assimilation and production optimization. PhD thesis Stanford University, 2013.

[2]. M. Heyouni, K. Jbilou. Matrix Krylov subspace methods for large scale model reduction problems.Appl. Math. Comput. $181,1215-1228,2006$.

[3]. T. Heijn, R. Markovinovi_c and J. D. Jansen.Generation of low-order reservoir models using system-theoretical concepts. SPE Journal, 2004, 9(2):202-218.

[4]. J. F. M. van Doren, R. Markovinovi ` c, and J. D. Jansen.Reduced-order optimal control of water flooding using proper orthogonal decomposition. Computational Geosciences,2006, 10:137-158.

[5]. M. A. Cardoso,L. J. Durlofsky,and P. Sarma.Development and application of reduced-order modeling procedures for subsurface flow simulation. International Journal for Numerical Methods in Engineering,2009, 77(9):1322-1350.

[6]. M. J. Rewienski. A Trajectory Piecewise-Linear Approach to Model Order Reduction of Nonlinear Dynamical Systems. PhD thesis, Massachusetts Institute of Technology, 2003.

[7]. Khalid Aziz, Antonin Settari. Petroleum Reservoir Simulation [M]. London: Applied Science Publishers, 1979:128-133.

[8]. J. D. Jansen. Systems Description of Flow Through Porous Media [M].Springer: Springer Briefs in Earth Sciences, 2013:21-36. 